\title{
Subtype of intraductal papillary mucinous neoplasm of the pancreas is important to the development of metachronous high-risk lesions after pancreatectomy
}

\author{
Ji Eun Kwon', Kee-Taek Jang², Youngju Ryu', Naru Kim¹, Sang Hyun Shin', \\ Jin Seok $\mathrm{Heo}^{1}$, Dong Wook Choi ${ }^{1}$, and In Woong Han ${ }^{1}$
}

\begin{abstract}
${ }^{1}$ Division of Hepatobiliary-Pancreatic Surgery, Department of Surgery, ${ }^{2}$ Department of Pathology and Translational Genomics, Samsung Medical Center, Sungkyunkwan University College of Medicine, Seoul, Korea
\end{abstract}

\begin{abstract}
Backgrounds/Aims: Although intraductal papillary mucinous neoplasm (IPMN) has showed a favorable prognosis compared to pancreatic ductal adenocarcinoma, its recurrence patterns have somewhat questionable in detail. After partial pancreatectomy for IPMN, the evaluation for risk of metachronous occurrence of high-risk lesions (HRL) in the residual pancreas is important to establish a postoperative surveillance modality and duration of follow-up. This study aimed to evaluate the factors that may predict the metachronous occurrence of HRL in the remnant pancreas after surgery of the IPMN. Methods: From 2005 to 2016, clinicopathologic and surveillance data for 346 consecutive patients who underwent surgical resection for IPMN were reviewed retrospectively. Histologic subtype was classified as gastric, intestinal, pancreato-biliary, or oncocytic type. Results: All of IPMN were classified as main duct $(n=64,18.5 \%)$, branch duct $(n=171,49.4 \%)$, and mixed type $(n=111,32.1 \%)$. Forty-eight patients $(13.9 \%)$ experienced recurrence during follow-up. Among these, 9 patients $(2.6 \%)$ were identified to metachronous development of HRL in the remnant pancreas. After multivariate analysis, high-grade dysplasia (HGD) or invasive carcinoma (IC) compared to low- or intermediate dysplasia was only independent risk factor for recurrence ( $\mathrm{HR} 3.688,95 \% \mathrm{Cl} 2.124-12.524, p=0.009)$. The independent risk factors for metachronous development were HGD/IC (HR 8.414, 95\% Cl 4.310- 16.426, $p=0.001$ ), and intestinal/ pancreato-biliary subtype compared to gastric subtype (HR 7.874, 95\% Cl 3.650- 27.027, $p=0.010)$. Conclusions: Patients with high-grade dysplasia or invasive carcinoma, and with intestinal or pancreatobiliary subtype should undergo close, long-term surveillance of the remnant pancreas after initial resection. (Ann Hepatobiliary Pancreat Surg 2019;23: 365-371)
\end{abstract}

Key Words: Intraductal papillary mucinous neoplasm; Pancreatectomy; Remnant pancreas; Subtype; Metachronous

\section{INTRODUCTION}

Intraductal papillary mucinous neoplasm (IPMN) is a cystic precursor of pancreatic cancer characterized by dilated main and/or branch ducts with papillary projections composed of dysplastic mucinous epithelium. ${ }^{1-4}$ IPMN has a wide histological spectrum ranging from low- (LGD), intermediate-(IGD), high-grade dysplasia (HGD) to invasive carcinoma (IC), and the management of IPMN continues to evolve. ${ }^{1-4}$

IPMNs have unique features, and multiple sites of occurrence in the same pancreas are often observed. IPMN probably represents a pancreatic "field defect", which means all pancreatic ductal epithelial cells are at risk of dysplastic change, and this can be apparent in patients with multifocal BD-IPMNs. ${ }^{2,4}$ Also, it is recognized that a proportion of resected patients for IPMN evolve over time and can become metachronous IPMN that patients are at an increased risk of developing conventional pancreatic ductal adenocarcinoma (PDAC) elsewhere in the residual gland. Because of this, all patients with IPMN, including even those with non-invasive IPMN with negative surgical margin, should undergo surveillance after resection to detect the development of a new IPMN re-

Received: July 25, 2019; Accepted: September 26, 2019

Corresponding author: In Woong Han

Division of Hepatobiliary-Pancreatic Surgery, Department of Surgery, Samsung Medical Center, Sungkyunkwan University School of Medicine, 81 Irwon-ro, Gangnam-gu, Seoul 06351, Korea

Tel: +82-2-3410-0772, Fax: +82-2-3410-6980, E-mail: cardioman76@gmail.com

Copyright (C) 2019 by The Korean Association of Hepato-Biliary-Pancreatic Surgery

This is an Open Access article distributed under the terms of the Creative Commons Attribution Non-Commercial License (http://creativecommons.org/ censes/by-nc/4.0) which permits unrestricted non-commercial use, distribution, and reproduction in any medium, provided the original work is properly cited. Annals of Hepato-Biliary-Pancreatic Surgery • pISSN: 2508-5778 - elSSN: 2508-5859 
quiring surgery or concomitant PDAC. ${ }^{4-7}$ However, the risk factors and characteristics of metachronous occurrence of IPMNs in the remnant pancreas have been unclear. As a result, there has been still debated the details for follow-up, such as, indication, methods, interval, and timing of follow-up. ${ }^{2-4}$

The cell lineage of the "papillary component" of IPMNs, such as, gastric, intestinal, pancreatobiliary, and oncocytic forms has been known clinicopathologic significance. ${ }^{2,4,8-11}$ The prognostic significance of these subtype showed heterogeneous results for recurrences and metachronous IPMN development in remnant pancreas after initial partial pancreatectomy. ${ }^{6,9-11}$ Thus, the aim of this study was to identify the factors including these subtypes that predict recurrences and metachronous occurrence high-risk lesions (HRL) in the remnant pancreas after partial pancreatectomy for IPMN, and to make comprehensive follow-up strategy after resection of IPMN.

\section{PATIENTS AND METHODS}

After our institutional review board approval (No. 2017-07-016-005), clinicopathologic and surveillance data of consecutive 346 patients who underwent pancreatectomy for IPMN at the Samsung Medical Center from January 2005 to December 2016 were reviewed retrospectively from prospectively maintained electronic database system

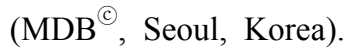

\section{Surgical indication}

Before 2014, surgical indications followed International Association of Pancreatology (IAP) Sendai guidelines in 2006, ${ }^{1}$ including main duct type IPMNs, branch duct type IPMNs with cysts larger than $30 \mathrm{~mm}$, main pancreatic duct dilatation exceeding $5 \mathrm{~mm}$, the appearance of new mural nodules, or the presence of any symptoms. Surgery was also performed when cysts showed significant growth or when there was increased suspicion of malignancy. From 2014 to 2016, surgical indication was modified according to IAP Fukuoka guidelines in $2012,{ }^{2}$ and were categorized as 'high risk stigmata', that are 'obstructive jaundice in a patient with cystic lesion of the head of the pancreas', 'enhancing solid component within cyst', and 'main pancreatic duct $>10 \mathrm{~mm}$ in size'.

\section{Surgical procedure}

Surgical procedures, such as, pancreaticoduodenectomy, central pancreatectomy or distal pancreatectomy, were determined according to the location and extent of the IPMN. If no invasive lesions were detected preoperatively, then laparoscopic procedures or limited resection were undertaken. In case of multifocal lesions, lesions without highrisk indications were not resected, in accordance with the 2006 IAP Sendai guidelines and Fukuoka guidelines in 2012. ${ }^{1,2}$ Most of pancreatic resection margins were accessed intraoperatively using frozen sections. Additional pancreatic resection or completion total pancreatectomy was done if HGD or IC was recognized.

\section{Postoperative surveillance}

Postoperative follow up based on radiologic examination and blood test including tumor markers was performed every 3 to 6 months. Radiologic examination, such as Computed Tomography (CT) or Magnetic Resonance Imaging (MRI) was carried out every 3 months during the initial 1 to 2 years after surgery for invasive IPMN and/or concomitant PDAC, and every 6 months thereafter. Additional endoscopic ultrasonography and/or endoscopic retrograde pancreatography were conducted in case of abnormal findings such as elevation of tumor markers, worsening diabetes mellitus, presence of new lesions, dilation of the main pancreatic duct, or morphological changes in residual IPMNs. After 2014, the 2012 IAP Fukuoka surveillance guidelines have been followed. ${ }^{2}$

\section{Histologic classifications of IPMN}

Pancreas specimens were serially sectioned at $5 \mathrm{~mm}$ intervals. The degree of dysplasia was classified to four distinct categories, such as, LGD, IGD, HGD, and IC. Also, all of IPMNs were subclassified as gastric, intestinal, pancreatobiliary, and oncocytic type. ${ }^{8-10}$ All of surgical specimen were reviewed by single pancreas-specialized pathologist with over 20 years of experiences to determine subtypes of IPMN.

\section{Definition and recurrence or metachronous high risk lesion}

Tumor recurrence detected with CT or MRI was confirmed by biopsy if possible. New tumors including remnant pancreas, resection margin, peri-pancreatic area, or 
systemic metastasis were included recurrences. However, recurrences resulting from malignancies in other organs were not included in the analysis. Remnant pancreas were thoroughly examined, and the occurrence of new IPMNs was monitored. Metachronous HRL was defined by all newly occurring IPMNs in the remnant pancreas except resection margin, which was examined histologically as HGD or IC. In non-resected cases, HRLs were diagnosed by cytological examination or radiological findings, which strongly suggested malignancy according to IAP guidelines. ${ }^{1,2,4}$

\section{Statistical analysis}

The Kaplan-Meier method was used to estimate survival. Variables that were significant in univariate analysis were analyzed by multiple regression analysis using the Cox proportional hazards regression model to determine independent predictive factors. Two-sided $p$-value less than 0.05 was considered statistically significant. All statistical analyses were conducted using SPSS 23.

\section{RESULTS}

\section{Clinicopathologic characteristics}

Demographic findings of the study are shown in Table 1. Mean age of patients was 63.2 and $70 \%$ of patients were male. All of IPMN were classified as main duct $(n=64$, $18.5 \%)$, branch duct $(n=171,49.4 \%)$, and mixed type $(n=$ $111,32.1 \%)$. Forty-eight patients $(13.9 \%)$ experienced recurrence during follow-up. Among these, 9 patients (3.0\%) were identified to metachronous development of HRL in the remnant pancreas (Table 1).

\section{Risk factors analysis for recurrences after initial surgery}

Patients with HGD/IC had significantly lower 5-year disease-free survival (5Y DFS) than among those with LGD/ IGD (5Y DFS 69.5\% vs. 90.1\%, $p=0.008$; Fig. 1A). Also, the patients with intestinal or pancreatobiliary subtype IPMNs had significantly lower DFS than those with gastric subtype IPMNs (5Y DFS $71.1 \%$ vs. $89.4 \%, p=$ 0.001; Fig. 1B). After univariate analysis, high serum CA 19-9 level (HR 2.941, 95\% CI 1.028- 8.416, $p=0.048$ ), cyst size larger than $3 \mathrm{~cm}$ (HR 2.568, 95\% CI 1.035- 4.953, $p=0.042$ ), HGD/IC compared to LGD/IGD (HR 4.624, 95\%
Table 1. Clinicopathological features of 346 resected IPMN

\begin{tabular}{|c|c|}
\hline Demographics, $n=346$ & $\mathrm{~N}, \%$ or range \\
\hline Age, mean, years (SD) & $63.1(9.2)$ \\
\hline Gender (male:female) & $234: 112$ \\
\hline Body mass index, mean, $\mathrm{kg} / \mathrm{m}^{2}$ (SD) & $23.7(2.9)$ \\
\hline CEA, median (range) & $1.7(0.05-119.5)$ \\
\hline CA19-9, median (range) & $12.0(0.2-43984)$ \\
\hline \multicolumn{2}{|l|}{ IPMN type } \\
\hline Main duct & $64(18.5)$ \\
\hline Branch duct & $171(49.4)$ \\
\hline Mixed & $111(32.1)$ \\
\hline \multicolumn{2}{|l|}{ Location } \\
\hline Head & $206(59.5)$ \\
\hline Body, tail & $96(27.7)$ \\
\hline Diffuse & $44(12.7)$ \\
\hline Cyst size, mean, cm (SD) & $3.43(1.8)$ \\
\hline \multicolumn{2}{|l|}{ Operation } \\
\hline pancreatoduodenectomy & $193(55.7)$ \\
\hline $\begin{array}{l}\text { Distal pancreatectomy } \\
\text { (with or without splenectomy) }\end{array}$ & $122(35.2)$ \\
\hline Total pancreatectomy & $44(12.7)$ \\
\hline Central pancreatectomy & $14(4.0)$ \\
\hline Enucleation & $13(3.7)$ \\
\hline \multicolumn{2}{|l|}{ Pathology } \\
\hline Low-grade dysplasia & $131(37.9)$ \\
\hline Intermediate-grade dysplasia & $107(30.9)$ \\
\hline High-grade dysplasia & $15(4.3)$ \\
\hline Invasive carcinoma & $93(26.8)$ \\
\hline $\begin{array}{l}\text { Subtype (Gastric/Intestinal/ } \\
\text { Pancreatobiliary/Oncocytic) }\end{array}$ & $225 / 75 / 29 / 5$ \\
\hline Metachronous high risk lesion* & $9(3.0)$ \\
\hline Recurrences & $48(13.9)$ \\
\hline
\end{tabular}

*Patients after total pancreatectomy were excluded by definition

CI 1.265- 14.002, $p=0.008)$, and intestinal/ pancreatobiliary subtype compared to gastric subtype (HR 3.135, 95\% CI 1.681- 5.848, $p=0.001)$ were identified risk factor for recurrence. However, HGD/IC was only independent risk factor for recurrence (HR 3.688, 95\% CI 2.124- 12.524, $p=0.009$ ) after multivariate analysis (Table 2).

\section{Risk factors analysis for metachronous development of high risk lesion}

After uni- and multivariate analysis, the independent risk factors for metachronous development of HRL were HGD/IC (HR 8.414, 95\% CI 4.310- 16.426, $p=0.001$ ), and intestinal/pancreatobiliary subtype compared to gastric subtype (HR 7.874, 95\% CI 3.650- 27.027, $p=0.010$ ) (Table 3). All of patients details who developed metachronous HRL were described in Table 4. 
A

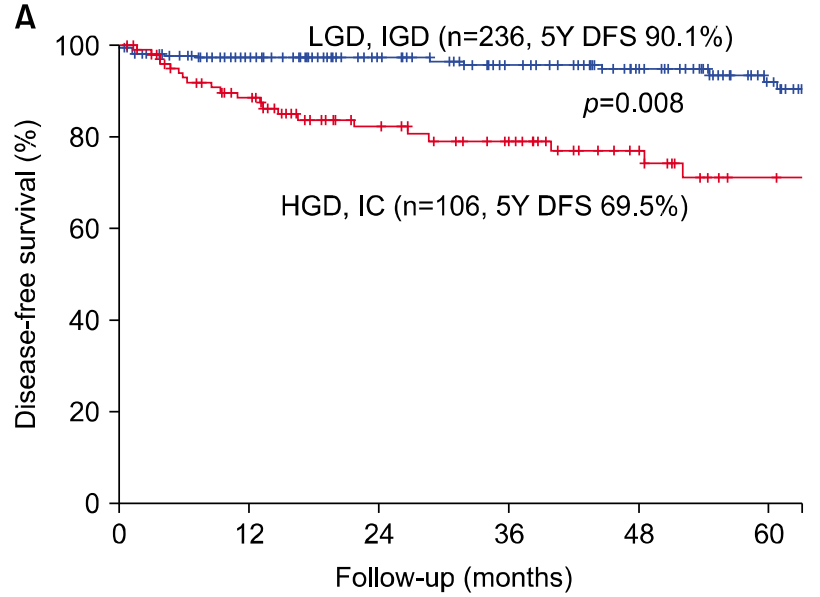

B

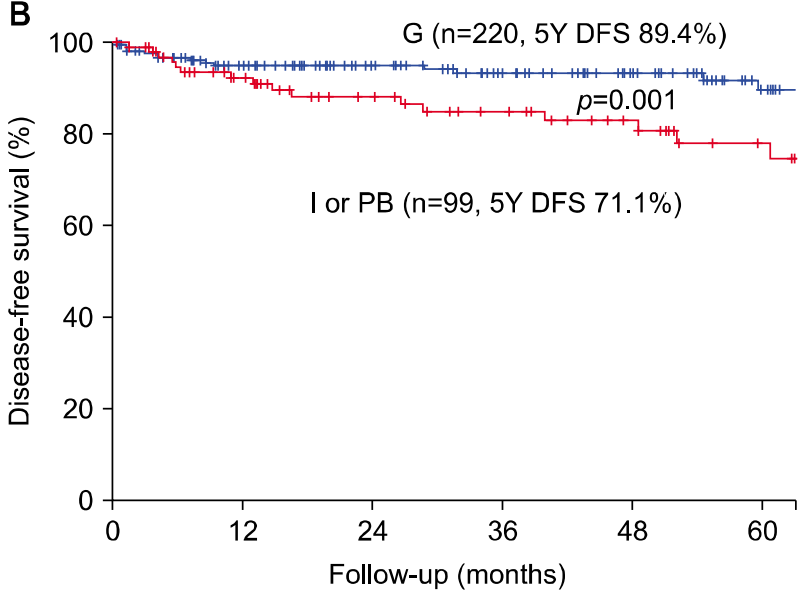

Fig. 1. Disease-free survival after pancreatectomy for IPMN. (A) DFS according to initial pathology. (B) DFS according to initial IPMN subtype. HGD, high-grade dysplasia; IGD, intermediate-grade dysplasia; LGD, low-grade dysplasia; IC, invasive carcinoma; 5Y DFS, 5-year disease-free survival; G, gastric subtype; I, intestinal subtype; PB, pancreato-biliary subtype.

Table 2. Risk factors analysis for recurrences after initial surgery

\begin{tabular}{|c|c|c|c|c|c|c|}
\hline \multirow{2}{*}{ Variables } & \multicolumn{3}{|c|}{ Univariate analysis } & \multicolumn{3}{|c|}{ Multivariate analysis } \\
\hline & HR & $95 \% \mathrm{CI}$ & $p$ & HR & $95 \% \mathrm{CI}$ & $p$ \\
\hline Female gender & 1.610 & $0.437-5.698$ & 0.344 & & & \\
\hline Age $(>65)$ & 1.253 & $0.823-4.574$ & 0.754 & & & \\
\hline Body Mass Index $(>25)$ & 2.106 & $0.756-4.238$ & 0.624 & & & \\
\hline Location (Body/tail vs. head) & 1.300 & $0.531-6.858$ & 0.358 & & & \\
\hline MD or mixed (vs. BD) & 1.683 & $0.435-6.073$ & 0.522 & & & \\
\hline $\mathrm{CEA}>5 \mathrm{ng} / \mathrm{ml}$ & 1.595 & $0.856-2.971$ & 0.142 & & & \\
\hline CA $19-9>37(\mathrm{U} / \mathrm{ml})$ & 2.941 & $1.028-8.416$ & 0.048 & 1.055 & $0.427-2.105$ & 0.896 \\
\hline Cyst size $>3 \mathrm{~cm}$ & 2.568 & $1.035-4.953$ & 0.042 & 1.969 & $0.816-4.021$ & 0.152 \\
\hline $\mathrm{HGD} / \mathrm{IC}$ vs. LGD/IGD & 4.624 & $1.265-14.002$ & 0.008 & 3.688 & $2.124-12.524$ & 0.009 \\
\hline Microscopic residual tumor (HGD/IC) & 1.227 & $0.741-10.677$ & 0.095 & 2.262 & $0.538-8.870$ & 0.238 \\
\hline $\mathrm{I} / \mathrm{PB}$ vs. $\mathrm{G}$ & 3.135 & $1.681-5.848$ & 0.001 & 1.175 & $0.978-4.667$ & 0.067 \\
\hline
\end{tabular}

HR, hazard ratio; CI, confidence interval; MD, main duct; $\mathrm{BD}$, branch duct; HGD, high-grade dysplasia; IGD, intermediate-grade dysplasia; LGD, low-grade dysplasia; IC, invasive carcinoma; G, gastric subtype; I, intestinal subtype; PB, pancreato-biliary subtype

Table 3. Risk factors analysis for metachronous development of high risk lesion

\begin{tabular}{|c|c|c|c|c|c|c|}
\hline \multirow{2}{*}{ Variables } & \multicolumn{3}{|c|}{ Univariate analysis } & \multicolumn{3}{|c|}{ Multivariate analysis } \\
\hline & HR & $95 \% \mathrm{CI}$ & $p$ & HR & $95 \% \mathrm{CI}$ & $p$ \\
\hline Female gender & 0.723 & $0.060-8.698$ & 0.796 & & & \\
\hline Age $(>65)$ & 1.353 & $0.623-6.574$ & 0.652 & & & \\
\hline Body mass index $(>25)$ & 1.632 & $0.485-1.965$ & 0.857 & & & \\
\hline Location (Body/tail vs. head) & 0.418 & $0.108-2.180$ & 0.055 & 0.521 & $0.881-4.027$ & 0.281 \\
\hline MD or mixed (vs. BD) & 1.875 & $0.435-6.073$ & 0.483 & & & \\
\hline $\mathrm{CEA}>5 \mathrm{ng} / \mathrm{ml}$ & 1.595 & $0.856-2.971$ & 0.142 & & & \\
\hline CA 19-9 >37 (U/ml) & 1.807 & $0.360-9.060$ & 0.365 & & & \\
\hline Cyst size $>3 \mathrm{~cm}$ & 1.598 & $0.956-3.267$ & 0.089 & & & \\
\hline HGD/IC vs. LGD/IGD & 3.980 & $1.697-9.057$ & 0.017 & 8.414 & $4.310-16.426$ & 0.001 \\
\hline Microscopic residual tumor (HGD/INV) & 3.259 & $0.354-10.489$ & 0.317 & & & \\
\hline $\mathrm{I} / \mathrm{PB}$ vs. $\mathrm{G}$ & 1.916 & $1.112-5.495$ & 0.009 & 7.874 & $3.650-27.027$ & 0.010 \\
\hline
\end{tabular}

HR, hazard ratio; CI, confidence interval; MD, main duct; BD, branch duct; HGD, high-grade dysplasia; IGD, intermediate-grade dysplasia; LGD, low-grade dysplasia; IC, invasive carcinoma; G, gastric subtype; I, intestinal subtype; PB, pancreato-biliary subtype 
Table 4. Patients details with metachronous high risk lesion

\begin{tabular}{|c|c|c|c|c|c|c|c|c|}
\hline Age/sex & $\begin{array}{c}\text { Initial } \\
\text { surgery }\end{array}$ & $\begin{array}{l}\text { Histologic } \\
\text { grade }\end{array}$ & Location & $\begin{array}{c}\text { Morphologic } \\
\text { type }\end{array}$ & Subtype & $\begin{array}{l}\text { DFS } \\
(\mathrm{mo})\end{array}$ & $\begin{array}{l}\text { Second } \\
\text { surgery }\end{array}$ & $\begin{array}{c}\text { Second } \\
\text { histologic grade }\end{array}$ \\
\hline $54 / \mathrm{F}$ & Central & IGD & Body & $\mathrm{BD}$ & - & 20.6 & - & - \\
\hline $72 / \mathrm{M}$ & SPDP & LGD & Tail & $\mathrm{BD}$ & G & 96.8 & - & - \\
\hline $63 / \mathrm{F}$ & $\mathrm{DP}$ & IC & Body & MD & I & 26.7 & Total & IC \\
\hline $47 / \mathrm{M}$ & DP & IC & Body to tail & Mixed & I & 55.4 & - & - \\
\hline $61 / \mathrm{M}$ & PPPD & IGD & Head & Mixed & I & 18.4 & - & - \\
\hline $57 / \mathrm{F}$ & SPDP & HGD & Tail & $\mathrm{BD}$ & I & 48.6 & - & - \\
\hline $50 / \mathrm{F}$ & $\mathrm{DP}$ & LGD & Body & MD & G & 34.2 & - & - \\
\hline $71 / \mathrm{M}$ & PPPD & HGD & Head & Mixed & $\mathrm{P}$ & 20.0 & - & - \\
\hline $64 / \mathrm{M}$ & PPPD & IGD & Head & $\mathrm{BD}$ & $\mathrm{G}$ & 12.6 & - & - \\
\hline
\end{tabular}

DFS, disease-free survival; MD, main duct; BD, branch duct; HGD, high-grade dysplasia; IGD, intermediate-grade dysplasia; LGD, low-grade dysplasia; IC, invasive carcinoma; G, gastric subtype; I, intestinal subtype; PB, pancreato-biliary subtype; SPDP, spleen-preserving distal pancreatectomy; DP, distal pancreatectomy; PPPD, pylorus-preserving pancreatoduodeectomy

\section{DISCUSSION}

It is now widely accepted that patients with IPMN who undergo partial pancreatectomy reportedly remain at risk of developing metachronous IPMN or concomitant PDAC. $5,7,11,12$ Moreover, some patients have secondary IPMNs with $\mathrm{HGD} / \mathrm{IC}$, which is an indication for resection, although the initial surgical margins were free from neoplastic lesions. ${ }^{11,13}$ However, the rate of new recurrence of metachronous IPMN following resection is difficult to determine from the literature, ${ }^{2}$ because MPD dilation in the distal pancreas following resection may be secondary to anastomotic stenosis or caused by true IPMN recurrence, and better imaging in the postoperative setting may reveal a previously undetected IPMN. Because of these limitations, the recurrence rates in the remnant gland were reported to be $0-20 \%,{ }^{2,5,11,13,14}$ but the characteristics of patients harboring such lesions have not been well assessed.

In this study, intestinal or pancreatobiliary subtype is one of the independent risk factors for metachronous development compared to gastric subtype (Table 3 ). It is well known that intestinal subtype IPMNs can have invasive carcinoma with relatively indolent behavior. ${ }^{2,8}$ Also, pancreatobiliary type is regarded by some as an aggressive and high-grade version of the gastric type. ${ }^{2,4,8}$ There was a similar report that partial pancreatectomy for pancreatobiliary subtype of IPMN was a predictor for the metachronous development of concomitant PDAC in the pancreatic remnant. $^{6,11}$ However, Ideno et al. ${ }^{10}$ showed that IPMN having concomitant PDAC were frequently of gastric sub- type even regarded that gastric type is typically low grade, with only a small percentage developing into carcinoma. ${ }^{2,49}$ As a result, to determine the relationship for risk of metachronous development and subtype of IPMN, the larger population study such as nationwide- based cohort study will be needed.

From the point of view that a metachronous lesion is a kind of multifocality, there is no convincing evidence that the risk of invasive IPMN multiplies according to the number of lesions. In one study, patients with unifocal BD-IPMN carried a higher risk than those with multifocal BD-IPMNs, ${ }^{15}$ whereas another reported a higher rate of IC or HGD in multifocal BD-IPMNs. ${ }^{16}$ Also, Miyasaka et al. ${ }^{11}$ reported that initial $\mathrm{HGD} / \mathrm{IC}$ was an independent predictive factor for metachronous $\mathrm{HGD} / \mathrm{IC}$ in the remnant pancreas. In this study, $\mathrm{HGD} / \mathrm{IC}$ is one of the independent risk factors for metachronous development of HRL (Table 3). As a result, we think HGD/IC indicate that close attention should also be paid to the remnant pancreas for the possible development of metachronous HRL.

In this study, type of IPMN, such as, main duct or mixed type, was not a risk factor for metachronous development (Table 3). However, a recent molecular analysis demonstrated the possibility of monoclonal skip implantation in main duct type IPMN could cause potential metachronous lesions. ${ }^{17}$ Also, Kang et al. ${ }^{5}$ reported that the rate of recurrent IPMN in the remnant pancreas requiring surgical treatment was higher in main duct type than in branch duct type IPMNs. To find out this discrepancy, we 
have a plan to perform nationwide multicenter study to identify relationship between the type of IPMN and metachronous development.

Although invasive IPMN may be associated with a poor prognosis, the long-term outcome after resection of most cases of IPMN is globally better than that of conventional PDAC. In this study, Patients with HGD to IC had a significantly higher rate of recurrence (Table 2) and a lower DFS rate (Fig. 1A) than patients with LGD to IGD in agreement with previous results. ${ }^{5,18}$ There were some studies that the subtype of IPMN appears to be an independent predictor of patient prognosis that intestinal or pancreatobiliary type IPMN showed poor survival than gastric type IPMN. ${ }^{4,8}$ However, in this study, the subtype of IPMN was even correlated with the DFS rate (Fig. 1B), but multivariate analysis revealed that there was no correlation between the subtype of IPMN and recurrence rate (Table 2).

The decision to follow an IPMN is a matter of clinical judgment based on the patient age, family history, symptoms, comorbidities, perceived pancreatic cancer risk, and patient preference. There is still controversy in the literature to guide the frequency and type of surveillance for IPMNs. ${ }^{1-4}$ American Gastroenterological Association guidelines $^{3}$ in 2015 restricted indications for surgery more stringently and recommended physicians to stop surveillance if no significant change had occurred in a pancreatic cyst after five years of surveillance, or if a patient underwent resection and a nonmalignant IPMN was found. However, there are little good long-term data to indicate whether surveillance can be safely discontinued after long-term stability. $^{2}$ On the contrary, most of studies suggested that long-term surveillance in patients with IPMN should be necessary and important because of the potential for secondary or recurrent development of HRL, such as concomitant PDAC. ${ }^{2,45,7,11}$ These metachronous lesions often develop more than 5-10 years after initial operation and the cumulative 5-year incidence of the development of concomitant HRL ranges from 2.2 to $8.8 \% .^{4-7,11,12,19-21}$ In this study, one patients experienced metachronous HRL after 96.8 months from initial distal pancreatectomy (Table 4). As a result, we think surveillance should continue as long as the patient remains fit for surgery, especially for patient with HGD/IC, intestinal/pancreatobiliary subtype of IPMN.

This study has some limitation. At first, we identified 9 metachronous HRL after initial surgery. Only one pa- tient received secondary completion total pancreatectomy who revealed with IC. The other 8 patients were strongly suspicious for HRL, such as, main pancreatic duct dilatation or enhancing mural nodule in CT or MRI but not conformed histologically because of unfit or refusal for secondary surgery. At second, we excluded oncocytic subtype for analysis as risk factor for metachronous development because of very small population $(n=5$, Table 1$)$ and no patient with oncocytic subtype developed metachronous lesion (Table 4). A larger study population is necessary to clarify the relationship between oncocytic subtype and metachronous development.

In conclusion, the risk of recurrence and metachronous development of high risk lesion does not diminish over time following resection, and surveillance should continue indefinitely as long as the patient remains fit for surgery. Also, special attention should be paid to the remnant pancreas in case of initial pathology with high grade dysplasia/ invasive carcinoma, and intestinal/ pancreatobiliary subtype.

\section{ACKNOWLEDGEMENTS}

The authors would like to thank Hyemin Kim (data manager, Department of Surgery, Samsung Medical Center, Sungkyunkwan University School of Medicine) for help with data collection.

\section{REFERENCES}

1. Tanaka M, Chari S, Adsay V, Fernandez-del Castillo C, Falconi M, Shimizu M, et al.; International Association of Pancreatology. International consensus guidelines for management of intraductal papillary mucinous neoplasms and mucinous cystic neoplasms of the pancreas. Pancreatology 2006;6:17-32.

2. Tanaka M, Fernández-del Castillo C, Adsay V, Chari S, Falconi M, Jang JY, et al.; International Association of Pancreatology. International consensus guidelines 2012 for the management of IPMN and MCN of the pancreas. Pancreatology 2012;12:183-197.

3. Vege SS, Ziring B, Jain R, Moayyedi P; Clinical Guidelines Committee, American Gastroenterology Association. American gastroenterological association institute guideline on the diagnosis and management of asymptomatic neoplastic pancreatic cysts. Gastroenterology 2015;148:819-822; quiz e12-e13.

4. Tanaka M, Fernández-Del Castillo C, Kamisawa T, Jang JY, Levy P, Ohtsuka T, et al. Revisions of international consensus Fukuoka guidelines for the management of IPMN of the pancreas. Pancreatology 2017;17:738-753.

5. Kang MJ, Jang JY, Lee KB, Chang YR, Kwon W, Kim SW. Long-term prospective cohort study of patients undergoing pancreatectomy for intraductal papillary mucinous neoplasm of the 
pancreas: implications for postoperative surveillance. Ann Surg 2014;260:356-363.

6. Hirono S, Kawai M, Okada K, Miyazawa M, Shimizu A, Kitahata $\mathrm{Y}$, et al. Long-term surveillance is necessary after operative resection for intraductal papillary mucinous neoplasm of the pancreas. Surgery 2016;160:306-317.

7. Date K, Ohtsuka T, Nakamura S, Mochidome N, Mori Y, Miyasaka Y, et al. Surveillance of patients with intraductal papillary mucinous neoplasm with and without pancreatectomy with special reference to the incidence of concomitant pancreatic ductal adenocarcinoma. Surgery 2018;163:291-299.

8. Furukawa T, Hatori T, Fujita I, Yamamoto M, Kobayashi M, Ohike N, et al. Prognostic relevance of morphological types of intraductal papillary mucinous neoplasms of the pancreas. Gut 2011; 60:509-516.

9. Mino-Kenudson M, Fernández-del Castillo C, Baba Y, Valsangkar NP, Liss AS, Hsu M, et al. Prognosis of invasive intraductal papillary mucinous neoplasm depends on histological and precursor epithelial subtypes. Gut 2011;60:1712-1720.

10. Ideno N, Ohtsuka T, Kono H, Fujiwara K, Oda Y, Aishima S, et al. Intraductal papillary mucinous neoplasms of the pancreas with distinct pancreatic ductal adenocarcinomas are frequently of gastric subtype. Ann Surg 2013;258:141-151.

11. Miyasaka Y, Ohtsuka T, Tamura K, Mori Y, Shindo K, Yamada $\mathrm{D}$, et al. Predictive factors for the metachronous development of high-risk lesions in the remnant pancreas after partial pancreatectomy for intraductal papillary mucinous neoplasm. Ann Surg 2016;263:1180-1187.

12. Marchegiani G, Mino-Kenudson M, Ferrone CR, Morales-Oyarvide $\mathrm{V}$, Warshaw AL, Lillemoe KD, et al. Patterns of recurrence after resection of ipmn: who, when, and how? Ann Surg 2015;262: 1108-1114.

13. He J, Cameron JL, Ahuja N, Makary MA, Hirose K, Choti MA, et al. Is it necessary to follow patients after resection of a benign pancreatic intraductal papillary mucinous neoplasm? J Am Coll Surg 2013;216:657-665; discussion 665-657.

14. Niedergethmann M, Grützmann R, Hildenbrand R, Dittert D, Aramin N, Franz M, et al. Outcome of invasive and noninvasive intraductal papillary-mucinous neoplasms of the pancreas (IPMN): a 10-year experience. World J Surg 2008;32:2253-2260.

15. Schmidt CM, White PB, Waters JA, Yiannoutsos CT, Cummings OW, Baker M, et al. Intraductal papillary mucinous neoplasms: predictors of malignant and invasive pathology. Ann Surg 2007; 246:644-651; discussion 651-644.

16. Fritz S, Schirren M, Klauss M, Bergmann F, Hackert T, Hartwig $\mathrm{W}$, et al. Clinicopathologic characteristics of patients with resected multifocal intraductal papillary mucinous neoplasm of the pancreas. Surgery 2012;152(3 Suppl 1):S74-S80.

17. Date K, Ohtsuka T, Fujimoto T, Tamura K, Kimura H, Matsunaga $\mathrm{T}$, et al. Molecular evidence for monoclonal skip progression in main duct intraductal papillary mucinous neoplasms of the pancreas. Ann Surg 2017;265:969-977.

18. Passot G, Lebeau R, Hervieu V, Ponchon T, Pilleul F, Adham M. Recurrences after surgical resection of intraductal papillary mucinous neoplasm of the pancreas: a single-center study of recurrence predictive factors. Pancreas 2012;41:137-141.

19. Tanno S, Nakano Y, Koizumi K, Sugiyama Y, Nakamura K, Sasajima J, et al. Pancreatic ductal adenocarcinomas in long-term follow-up patients with branch duct intraductal papillary mucinous neoplasms. Pancreas 2010;39:36-40.

20. Ikeuchi N, Itoi T, Sofuni A, Itokawa F, Tsuchiya T, Kurihara T, et al. Prognosis of cancer with branch duct type IPMN of the pancreas. World J Gastroenterol 2010;16:1890-1895.

21. Sahora K, Ferrone CR, Brugge WR, Morales-Oyarvide V, Warshaw $\mathrm{AL}$, Lillemoe $\mathrm{KD}$, et al. Effects of comorbidities on outcomes of patients with intraductal papillary mucinous neoplasms. Clin Gastroenterol Hepatol 2015;13:1816-1823. 